\title{
First Spaceborne Demonstration of Digital Beamforming for Azimuth Ambiguity Suppression
}

\author{
Jung-Hyo Kim, Member, IEEE, Marwan Younis, Senior Member, IEEE, Pau Prats-Iraola, Member, IEEE, \\ Martina Gabele, and Gerhard Krieger, Senior Member, IEEE
}

\begin{abstract}
Over the past years, the use of multiple antenna apertures combined with digital beamforming (DBF) has been spotlighted as a promising solution for the fundamental restriction for high-resolution and wide-swath spaceborne synthetic aperture radar (SAR) imaging. In this paper, we present the first spaceborne experiment of a DBF technique on receive, using the TerraSAR-X dual receive antenna mode. For this experiment, we implemented a DBF module, which includes the reconstruction filter as a digital beam former and associated signal processing for calibration and channel balancing. The experimental results exhibit the successful ambiguity suppression capability of the DBF and validate the high potential of the DBF both for advanced future and current SAR systems.
\end{abstract}

Index Terms-Azimuth ambiguity suppression, digital beamforming (DBF), high-resolution wide-swath (HRWS) SAR imaging, reconstruction algorithm, synthetic aperture radar (SAR), TerraSAR-X dual receive antenna (DRA).

\section{INTRODUCTION}

A SYNTHETIC aperture radar (SAR) providing globalscale data with a fine spatial resolution plays an essential role in Earth observation and remote sensing. Modern SAR systems employing an analog beam-steering technique are capable of implementing multiple operation modes, such as the Stripmap, ScanSAR, and Spotlight mode, to fulfill the different requirements in terms of spatial resolution or coverage. Nevertheless, an inherent conflict between a wide coverage and a fine azimuth resolution has remained a complex issue to resolve in the state-of-the-art SAR concept. According to the minimum antenna area constraint [1], the ground range extent of the target region is limited by a given PRF, which corresponds to a spatial sampling rate in along-track. On the other hand, a high azimuth resolution can be achieved by processing a wide Doppler bandwidth signal, which requires a high sampling frequency, i.e., PRF. Consequently, the wideswath SAR imaging with a fine resolution is fundamentally a contradiction in conventional SAR imaging.

A multichannel SAR combined with digital beamforming (DBF) capability has received a great deal of attention as a

Manuscript received December 9, 2011; revised April 5, 2012; accepted May 11, 2012. Date of publication July 10, 2012; date of current version December 19, 2012

The authors are with the German Aerospace Center (DLR), Microwaves and Radar Institute, 82234 Oberpfaffenhofen, Germany (e-mail: junghyo.kim@dlr.de).

Color versions of one or more of the figures in this paper are available online at http://ieeexplore.ieee.org.

Digital Object Identifier 10.1109/TGRS.2012.2201947 promising concept to overcome the above limitation. This DBFbased system concept is not limited in the Stripmap mode but is extended in burst mode by combining with the ScanSAR and Terrain Observation with Progressive Scan modes [2]. The multiple independent receive channels arrayed in flight direction increase the number of spatial samples, and a digital filter bank, i.e., digital beam former, coherently processes the multichannel signals. The major role of the DBF in this process is to suppress azimuth ambiguities caused by the spectral aliasing of spatially subsampled data, and to recover a wide Doppler spectrum for nonuniformly sampled data. In particular, a DBF technique, which is called the reconstruction algorithm, enables to constantly suppress the ambiguity for a wide range of PRF variation [3]. This technique has been preliminarily verified in a laboratory experiment using a multichannel SAR demonstrator on the ground with respect to point scatterers [4], [5] and in an airborne experiment using DLR's F-SAR system based on a switching mode of multiple antennas [6], [7]. Through both experiments, the ambiguity suppression capability of the DBF technique has been successfully demonstrated. However, a spaceborne experiment regarding a wide complex inhomogeneous scene and complete wave propagation channel effects remained a necessary step to complete the experimental verification of this advanced technique.

TerraSAR-X is an operational advanced SAR satellite for scientific and commercial uses [8]. Its SAR instrument equipped with the split antenna allows the simultaneous acquisition of reflected signals from targets by the preceding and succeeding segments of the antenna separately, so-called dual receive antenna (DRA) mode [9]. Since 2009, TerraSAR-X DRA mode campaigns have been carried out, and we have performed the first spaceborne DBF experiment using specially commanded TerraSAR-X DRA mode data takes. This paper summarizes these experiments and shows the promising experimental results. The primary goal of the experiment is to verify the DBF algorithm in the frame of a practical spaceborne SAR scenario and evaluate the performance in comparison to the conventional mode. The experimental results demonstrate not only the capability of ambiguity suppression but also the feasibility to utilize the DBF to SAR systems in operation, such as TerraSAR-X and Radarsat-2, for the purpose of the performance improvement of SAR products in terms of either the azimuth ambiguity level or the coverage.

This paper begins with an overview of the TerraSAR-X DRA mode in Section II. Describing the system architecture 


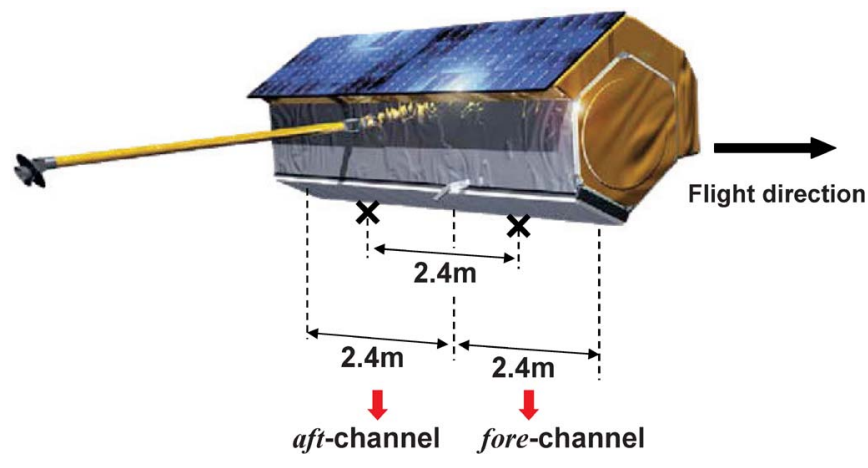

Fig. 1. TerraSAR-X DRA geometry. The baseline in along-track is defined by the difference between the phase centers of the two receive antennas.

and functions, this section links to the processing strategy adopted for this experiment and processing aspects in Section III. Sections IV and V present the experimental results and analyze them in detail, respectively. The final Section VI summarizes the implications of this experiment and discusses future perspectives.

\section{TERRASAR-X DRA Mode}

The DRA of the TerraSAR-X instrument is used for experimental data acquisitions in Stripmap mode only. The whole antenna $(4.8 \mathrm{~m})$ is electrically split into two halves $(2.4 \mathrm{~m}$ each). The preceding and the succeeding halves in flight direction are called the fore- and aft-channels, respectively. Fig. 1 depicts the DRA of TerraSAR-X and indicates the spatial baseline of $2.4 \mathrm{~m}$ between both channel phase centers. This spatial separation produces a phase difference exploited for applications such as moving target detection or ocean current studies as well as DBF [9]-[11]. Both the receive channels can be switched from pulse to pulse or simultaneously operated, depending on the experimental purposes [9]. The data from each channel are digitally sampled so that two independent data streams are available. For this DBF experiment, the entire antenna aperture is used for the pulse transmission, and both receive antenna halves are used for data collection at the same time.

Fig. 2 shows a block diagram of the hardware implementation of the TerraSAR-X DRA mode. Each segment of the antenna is composed of 192 array elements including individual transmit/receive (T/R) modules. The X-band hybrid coupler followed by the central electronics $(\mathrm{CE})$ unit combines the input fore- and aft-channel signals and produces the sum $(\Sigma)$ and the difference $(\Delta)$ channel signals, i.e.,

$$
\begin{aligned}
& r_{\Sigma}(t)=\frac{1}{\sqrt{2}} \cdot\left(s_{1}(t)+s_{2}(t)\right) \\
& r_{\Delta}(t)=\frac{1}{\sqrt{2}} \cdot\left(s_{1}(t)-s_{2}(t)\right)
\end{aligned}
$$

where $s_{1}(t)$ and $s_{2}(t)$ denote the fore- and the aft-channel signals, respectively. It is noted that the $\Sigma$-channel signal corresponds to the conventional single-antenna mode signal, since it uses the whole aperture as the final outcome. The use of the hybrid coupler allows to employ a redundant $\mathrm{CE}$ unit, which can replace the main $\mathrm{CE}$ in case of failure. In the conventional single-antenna mode, the $\Delta$-channel is disconnected from the

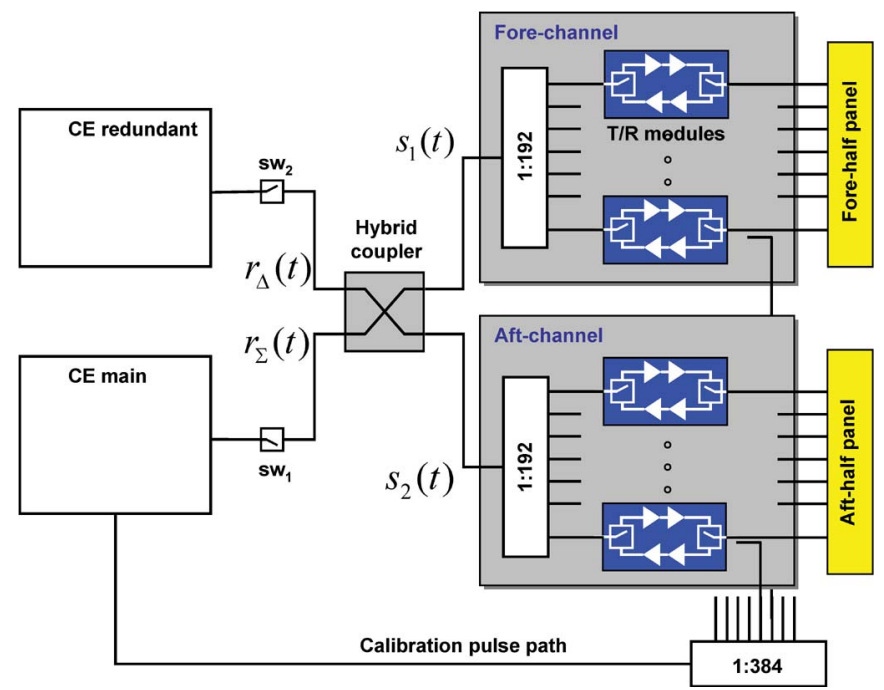

Fig. 2. Block diagram of TerraSAR-X DRA hardware implementation.

redundant CE by switching off (see Fig. 2), and only the $\Sigma$-channel signal is recorded. In contrast, both channel signals are stored in the DRA mode.

Although the TerraSAR-X system provides the dual-channel signals simultaneously acquired, the $\Sigma$ - and $\Delta$-channel signals are not directly applicable to the DBF experiment. Prior to the main DBF processing, the fore- and aft-channel data should be reconstructed. For this purpose, we implemented a DBF module, which includes several additional calibration and optimization steps. In the following section, we give an overview of the overall signal processing flow and describe the key processing steps in detail.

\section{Processing Overview}

The experimental processor used in this experiment is composed of two main parts: the DBF module and the conventional SAR processor. Fig. 3 shows the experimental processor schematic. The DBF module conducts several functionalities: a) the $\Sigma$ - and $\Delta$-channel calibration, b) the fore- and aft-channel reconstruction, c) the channel balancing of the reconstructed fore- and aft-channels, and d) the DBF based on the reconstruction algorithm. The first two steps are specific to the experiment using the specific TerraSAR-X DRA mode implementation, and the following two steps are common steps required in any DBF-based SAR system. The dedicated DBF modules have been integrated in the experimental TanDEM-X interferometric (TAXI) processor, which is a versatile tool developed at the Microwaves and Radar Institute, German Aerospace Center (DLR), to process monostatic and bistatic SAR data [12]. Its SAR processing unit produces SAR images on the basis of the extended chirp scaling (ECS) algorithm in the conventional single-antenna mode.

\section{A. $\Sigma$ - and $\Delta$-Channel Calibration}

In previous work, the calibration steps of the $\Sigma$ - and $\Delta$ channels were intensively studied, and the detailed procedures were described in [13]. From (1) and (2), the $\Sigma$ - and $\Delta$-channel signals produced by an ideal hybrid coupler can be added and 


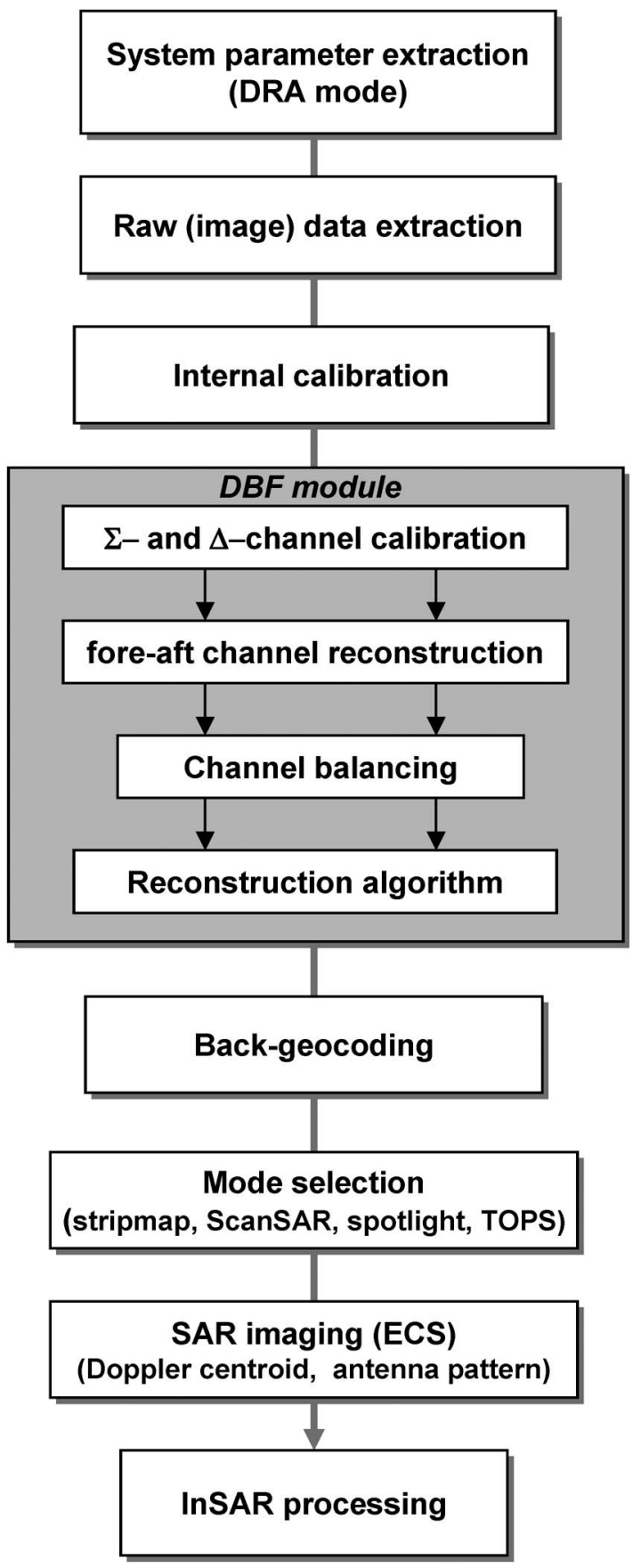

Fig. 3. Simplified block diagram of the DBF module integrated into the TAXI processor. The last processing step (InSAR processing) is not applied to data of the DBF experiment.

subtracted to recover the input fore- and aft-channel signals. However, due to imperfect characteristics, the hybrid coupler yields amplitude and phase offsets in the output signals, which cause a significant disturbance in the DBF as well as the foreand aft-channel reconstruction. The transfer function of the receiver can be described as a $2 \times 2$ matrix model, i.e.,

$\mathbf{H}_{\mathrm{Rx}}(f)=\frac{1}{\sqrt{2}} \cdot\left[\begin{array}{cc}\Delta g(f) \cdot \exp (j \cdot \Delta \varphi) & \Delta g(f) \cdot \exp (j \cdot \Delta \varphi) \\ 1 & -1\end{array}\right]$

where $\Delta g$ and $\Delta \varphi$ denote the frequency-dependent gain and phase offsets in the hybrid coupler, respectively, and $f$ stands for the frequency. In [13], it was shown that those offsets can be estimated out of the imaging signals and the receiver calibration pulses.

The TerraSAR-X system supports various calibration pulses in order to measure the system transfer functions of individual transmit and receive hardware. Therefore, the time-variant hardware characteristics can be compensated for each data take. The receive calibration beam for the standard DRA mode is used to estimate the amplitude offset $\Delta g$. The calibration pulse is shifted by $+\pi / 4$ and $-\pi / 4$ in phase for the $\Sigma$-channel and the $\Delta$-channel, respectively. This yields the $\pi / 2$ phase difference between both channels, and thereby, the calibration beam signals of each $\Sigma$ - and $\Delta$-channel are supposed to have the same amplitudes. The gain offset can be estimated by taking the average amplitude of $L$ calibration pulses, i.e.,

$$
\Delta g(f)=\sqrt{\frac{E\left\{R_{\Delta, \mathrm{cal}}(f, l) \cdot R_{\Delta, \mathrm{cal}}^{*}(f, l)\right\}}{E\left\{R_{\Sigma, \mathrm{cal}}(f, l) \cdot R_{\Sigma, \mathrm{cal}}^{*}(f, l)\right\}}}
$$

where $l=0,1,2, \ldots, L-1$, and $R_{\Sigma, \text { cal }}(f, l)$ and $R_{\Delta, \text { cal }}(f, l)$ denote the spectra of the calibration beams of the $\Sigma$ - and $\Delta$ channels, respectively. $E\{\cdot\}$ stands for the expectation value, and the asterisk $\left({ }^{*}\right)$ means the complex conjugate.

The phase offset $\Delta \varphi$ can be estimated from the interferometric phase between both channel Doppler spectra. In the ideal case, the Doppler spectra are given by [13]

$$
\begin{aligned}
& R_{\Sigma}\left(f_{\mathrm{D}}\right)=\sqrt{2} \cdot A\left(f_{\mathrm{D}}\right) \cdot \cos \left(\frac{\pi \cdot d}{2 \cdot v} \cdot f_{\mathrm{D}}\right) \\
& R_{\Delta}\left(f_{\mathrm{D}}\right)=j \cdot \sqrt{2} \cdot A\left(f_{\mathrm{D}}\right) \cdot \sin \left(\frac{\pi \cdot d}{2 \cdot v} \cdot f_{\mathrm{D}}\right)
\end{aligned}
$$

where $f_{\mathrm{D}}$ denotes the Doppler frequency, $d$ is the alongtrack baseline, $v$ indicates the sensor velocity, and $A\left(f_{\mathrm{D}}\right)$ is the azimuth antenna pattern in Doppler frequency. The interferometric phase of both channels is given by $\pi / 2$ for the negative Doppler frequencies and $-\pi / 2$ for positive Doppler frequencies, i.e.,

$$
\begin{aligned}
\angle\left(R_{\Sigma}\left(f_{\mathrm{D}}\right) \cdot R_{\Delta}^{*}\left(f_{\mathrm{D}}\right)\right) & =\angle\left(-j \cdot A^{2}\left(f_{\mathrm{D}}\right) \cdot \sin \left(\frac{\pi \cdot d}{v} \cdot f_{\mathrm{D}}\right)\right) \\
& = \begin{cases}\frac{\pi}{2} & \text { for }-1<\frac{d}{v} \cdot f_{\mathrm{D}}<0 \\
-\frac{\pi}{2} & \text { for } \quad 0<\frac{d}{v} \cdot f_{\mathrm{D}}<1 .\end{cases}
\end{aligned}
$$

The phase offset $\Delta \varphi$ is a difference between the ideal phase and a real measured phase. The above calibration approach is valid within the Doppler frequency range that satisfies the Nyquist sampling criterion.

\section{B. Fore- and Aft-Channel Reconstruction}

From the estimated gain and phase offsets, we have a complete knowledge about the transfer function matrix of the receiver in (3). Therefore, the fore- and aft-channels can be reconstructed by compensating the receiver transfer function, i.e.,

$$
\left[\begin{array}{l}
S_{1}(f) \\
S_{2}(f)
\end{array}\right]=\mathbf{H}_{\mathrm{Rx}}^{-1}(f) \cdot\left[\begin{array}{l}
R_{\Sigma}(f) \\
R_{\Delta}(f)
\end{array}\right]
$$


It may be noted that this simple transfer matrix model is sufficiently applicable to the $\Sigma$ - and $\Delta$-channel calibration, due to the sophisticated TerraSAR-X hardware.

\section{Channel Balancing}

The separated fore- and aft-channels possibly contain mismatches in amplitude and phase, which are not corrected in the previous calibration process and yield unknown array manifold effects on beamforming. The channel balancing described in this section removes such imbalances between both the reconstructed channels prior to the DBF. The importance of this channel balancing has been remarked in our preliminary experiments [4], [6].

The approach used in this experiment is based on the fact that the interferometric phase distribution between the two coregistered DRA channels has a mean value of zero ${ }^{1}$ [14]. A parameter that should be known in this method is hence the distance between the two antenna phase centers. Since the interferometric phase in the Doppler domain is caused by the antenna displacement, the distance can be estimated from

$$
\angle E\left\{S_{1}\left(f_{\mathrm{D}}\right) \cdot S_{2}^{*}\left(f_{\mathrm{D}}\right)\right\}=\psi_{\text {int }}\left(f_{\mathrm{D}}\right)=\frac{\pi \cdot d}{v} \cdot f_{\mathrm{D}}
$$

where we take the average in range-frequency in order to minimize the noise contribution. By normalizing the covariance, we obtain

$$
\sigma_{12}\left(f, f_{\mathrm{D}}\right)=\frac{E\left\{S_{1}\left(f, f_{\mathrm{D}}\right) \cdot S_{2}^{*}\left(f, f_{\mathrm{D}}\right)\right\}}{E\left\{\left|S_{2}\left(f, f_{\mathrm{D}}\right)\right|^{2}\right\}}
$$

where $E\left\{\sqrt{\left|\sigma_{12}\right|}\right\}$ is the amplitude imbalance, and $\angle \sigma_{12}$ is used to estimate the phase imbalance. Due to the coregistration, the phase distribution is concentrated around the zero phase. Thus, the shift of the phase histogram from zero corresponds to the phase imbalance [14]. In a practical implementation, a sliding average window was used in order to reduce the noise effect and improve the estimation accuracy.

\section{DBF-Reconstruction Algorithm}

The reconstruction algorithm finds optimum filter coefficients to suppress the azimuth ambiguity from a linear system of equations with respect to the antenna geometry and the SAR parameters [3]. The obtained filter is called the reconstruction filter, which effectively separates desired signals and ambiguity signals in the Doppler frequency domain. The reconstruction filter compensates for the phase shift resulting from both the antenna displacement and the spatial sampling, and thereby provides nearly steady performance even for nonuniformly sampled data.

Due to the linear property of the reconstruction filter, this DBF can be carried out at any stage in the experimental processor. According to the signal model in [3] and [15], the multichannel system can be regarded as a monostatic SAR that

\footnotetext{
${ }^{1}$ To avoid the influence of ocean current on the interferometric phase, data over the land area are only used in this approach.
}

is followed by additional time and phase shifts for each receive channel. In the case of a dual-channel system, the transfer function matrix for these additional shifts is described as

$$
\mathbf{H}_{\mathrm{Ch}}\left(f_{\mathrm{D}}\right)=\left[\begin{array}{ll}
H_{11}\left(f_{\mathrm{D}}\right) & H_{12}\left(f_{\mathrm{D}}+f_{\mathrm{PRF}}\right) \\
H_{21}\left(f_{\mathrm{D}}\right) & H_{22}\left(f_{\mathrm{D}}+f_{\mathrm{PRF}}\right)
\end{array}\right]
$$

where

$$
\begin{aligned}
H_{n m}\left(f_{\mathrm{D}}\right)=\exp \left(j \cdot \frac { \pi } { 2 v } \cdot d \cdot \left(f_{\mathrm{D}}\right.\right. & +(m-1) \\
& \left.\left.\cdot f_{\mathrm{PRF}}\right) \cdot(-1)^{n+1}\right)
\end{aligned}
$$

for $n, m=1,2$, and $f_{\mathrm{PRF}}$ denotes the PRF. It is noted that the amplitude pattern can be ignored in (12) since it is possible to incorporate the transfer function due to the antenna pattern into the monostatic SAR response [3]. The reconstruction filter matrix $\mathbf{P}$ is given by the inverse of the channel transfer function matrix, i.e.,

$$
\mathbf{P}=\mathbf{H}_{\mathrm{Ch}}^{-1} .
$$

For the TerraSAR-X DRA mode, the reconstruction filter matrix is derived using (11)-(13) as follows:

$$
\begin{aligned}
& P_{n m}\left(f_{\mathrm{D}}\right)= \frac{(-1)^{n-m}}{\exp \left(-j \cdot \frac{\pi}{2 v} \cdot d \cdot f_{\mathrm{PRF}}\right)-\exp \left(j \cdot \frac{\pi}{2 v} \cdot d \cdot f_{\mathrm{PRF}}\right)} \\
& \cdot \exp \left(j \cdot \frac { \pi } { 2 v } \cdot d \cdot \left(f_{\mathrm{D}}+\left(2^{n-1}-2(n-1)\right)\right.\right. \\
&\left.\left.\cdot f_{\mathrm{PRF}}\right) \cdot(-1)^{m}\right) \cdot
\end{aligned}
$$

In the case of a single-platform system, the antenna displacement $d$ is a constant, and the sampling rate $f_{\mathrm{PRF}}$ is only a variable for each data take. The reconstruction filtering deals with band-limited signals within the given PRF, so that this DBF process is completed by concatenating the band-limited signals in the Doppler domain, in order to reconstruct a wide aliasing-free Doppler spectrum, as illustrated in Fig. 4.

\section{DBF Experiment Using TerRaSAR-X DRA Mode}

The TerraSAR-X DRA mode campaigns have been performed over different locations with various PRFs, providing opportunities for several scientific experiments. This section starts with the description of the data takes used in this experiment, and in the following sections, experimental results are presented in detail.

\section{A. Data Acquisition}

In this paper, we present experimental results using two TerraSAR-X data takes, which are acquired over two different regions with different SAR parameters. Table I summarizes the parameters of the data takes (DT).

A common geographic aspect of both data takes is that these include wide sea clutter area, where ambiguities are more obviously visible. Another important point is the PRF and its corresponding spatial sampling distance. With respect to the velocity of the TerraSAR-X satellite $(7.65 \mathrm{~km} / \mathrm{s})$, a PRF required for the uniform sampling is approximately $3.187 \mathrm{kHz}(100 \%$ 

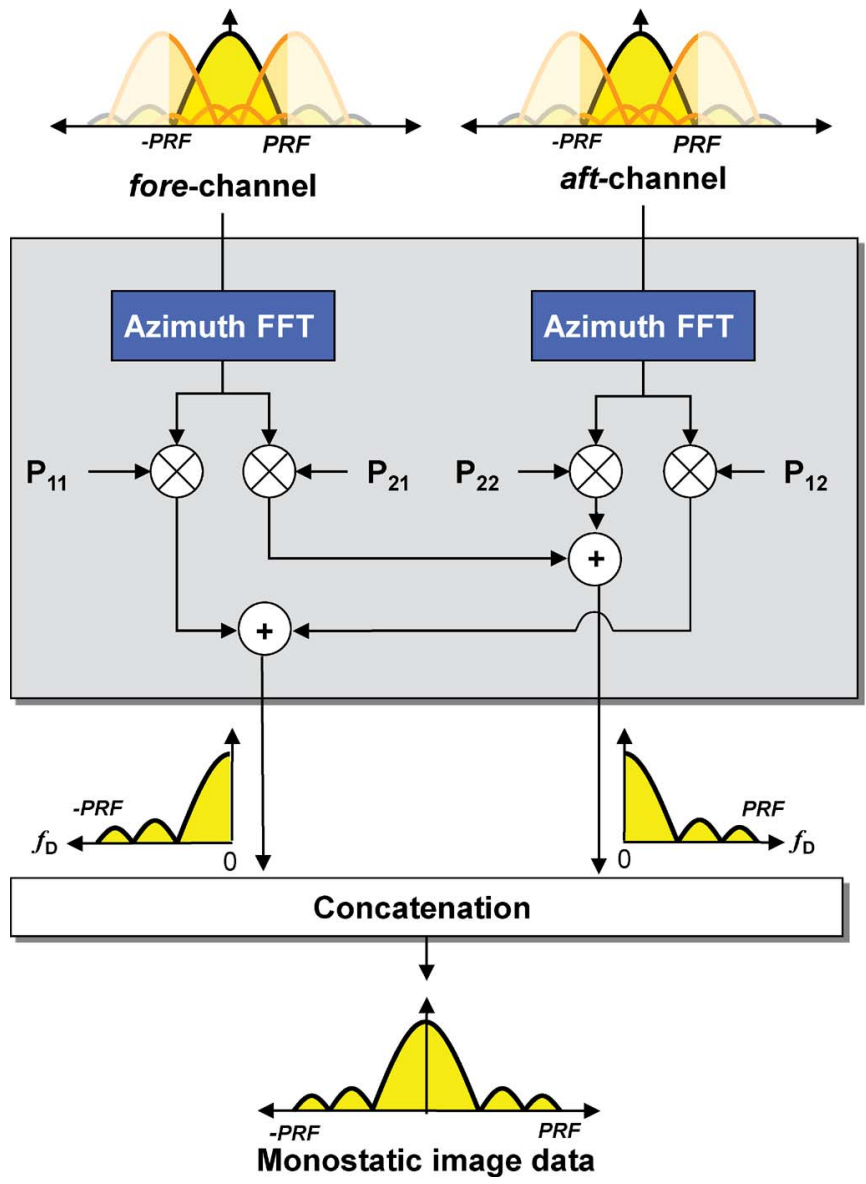

Fig. 4. Reconstruction filtering and the concatenation of the output signals for the TerraSAR-X system. Each output signal bandwidth is equivalent to the PRF, and after the concatenation, the signal bandwidth is increased by a factor of 2 in this case.

TABLE I

PARAMETERS OF THE ACQUiRed Data TAKes

\begin{tabular}{|c||c|c|}
\hline Parameters & Data take 1 & Data take 2 \\
\hline Acq. date & 25.04 .2009 & 24.03 .2010 \\
Time (UTC) & $06: 02: 36$ & $04: 39: 34$ \\
Duration & $8.76 \mathrm{sec}$ & $15.81 \mathrm{sec}$ \\
Region & Barcelona, Spain & Hawaii, USA \\
PRF & $3.773 \mathrm{kHz}$ & $2.852 \mathrm{kHz}$ \\
Uniformity & $118.4 \%$ & $89.5 \%$ \\
Chrip BW & $150 \mathrm{MHz}$ & $100 \mathrm{MHz}$ \\
Chirp length & $47.7 \mu \mathrm{sec}$ & $63.1 \mu \mathrm{sec}$ \\
Polarization & HH & HH \\
Mode & Stripmap & Stripmap \\
\hline
\end{tabular}

uniformity). In Fig. 5, the spatial sampling of the acquired data takes is depicted together with the uniform sampling. Fig. 5(a) shows the uniform sampling in the TerraSAR-X DRA mode. When the separation between two successive spatial samples corresponds to $1.2 \mathrm{~m}$, the sampling is uniform. On the other hand, the acquired data takes are nonuniformly sampled as shown in Fig. 5(b) and (c).

The 3.773-kHz PRF of DT1 leads to a separation of $0.827 \mathrm{~m}$ (118.4\% uniformity) between successive sample pairs, and in DT2, the sample separation becomes $1.48 \mathrm{~m}$ (89.5\% uniformity). Therefore, the data takes contain strong nonuniformity. The use of these nonuniformly sampled data aims at demonstrating the capability of the reconstruction algorithm.

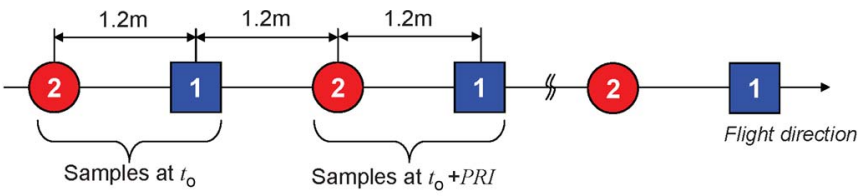

(a)

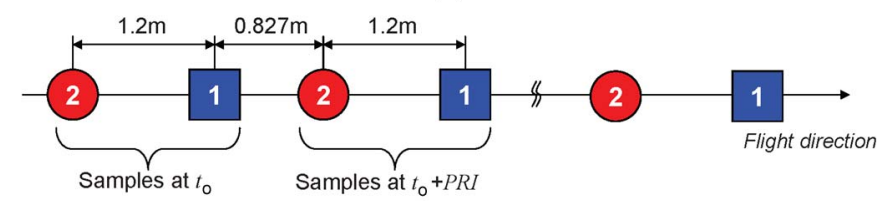

(b)

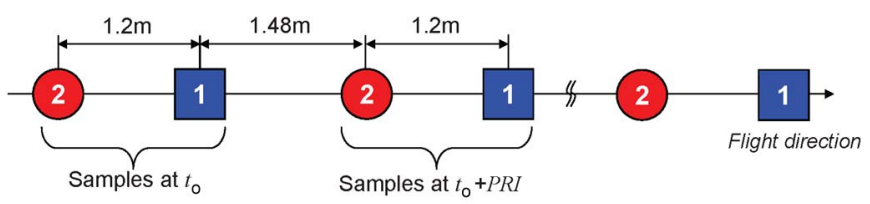

(c)

Fig. 5. Spatial data sampling for (a) the uniform sampling (3.187-kHz PRF), (b) DT1, and (c) DT2.

Section V will compare the imaging results by the reconstruction algorithm and simple data interleaving.

\section{B. Calibration and Channel Balancing}

Here, we present the calibration and the channel balancing results of the experimental data takes. As introduced in Section III-A and C, these processes are indispensable steps in order to perform the DBF. First, the $\Sigma$ - and $\Delta$-channels are synchronized in amplitude and phase. The amplitude offset between these channels was measured using internal calibration pulses, and the phase offset was estimated using image data. Fig. 6 exhibits the measured phase patterns before and after the calibration.

Due to constant phase offsets in both data takes, the phase patterns are shifted from the theoretical phase pattern, indicated by the dashed line in these figures. A remarkable aspect in this calibration process is that the phase pattern is affected by the PRF. The phase pattern of DT1 is analogous to the theoretical phase pattern, whereas that of DT2 is slightly distorted due to the spectral aliasing resulting from its low PRF $(2.852 \mathrm{kHz})$. Therefore, for appropriate calibration, a high PRF is required.

From the calibrated $\Sigma$ - and $\Delta$-channel data, we reconstructed the fore- and aft-channel data, which are eventually applied to the DBF. Frequency-dependent amplitude balancing compensates for the amplitude difference between the fore- and aftchannels in the 2-D frequency domain. The phase imbalance is estimated by measuring the offset of the interferometric phase distribution of coregistered data sets, according to the method in Section III-C. Fig. 6(c) and (f) presents the measured phase histograms.

It may be worth noting that the broad phase histogram of DT2 in Fig. 6(f) is due to the influence of the aliasing. However, this histogram provides a satisfactory estimate of phase imbalance, based on the stationary scene [16]. The measurement shows that a constant phase imbalance of $5.98^{\circ}$ and $8.79^{\circ}$ is contained in DT1 and DT2, respectively. Table II summarizes the measured 


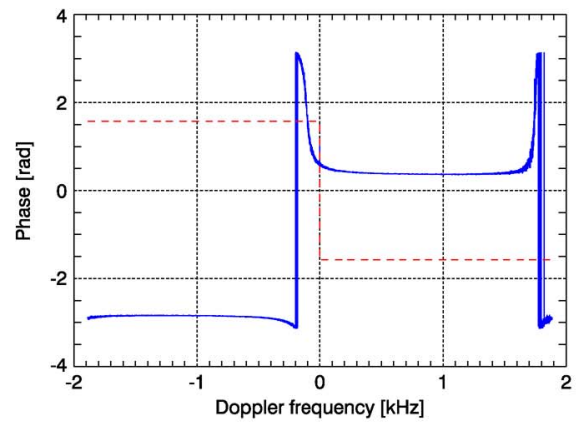

(a)

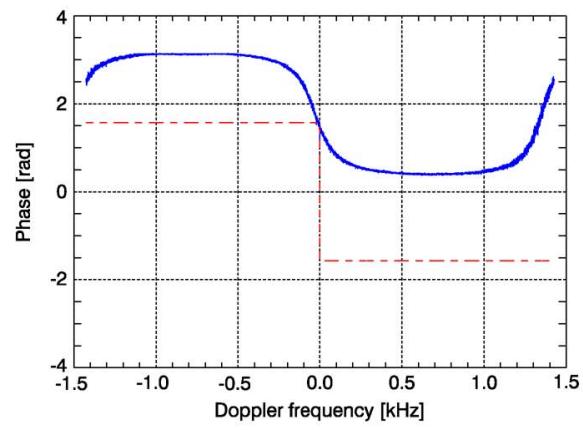

(d)

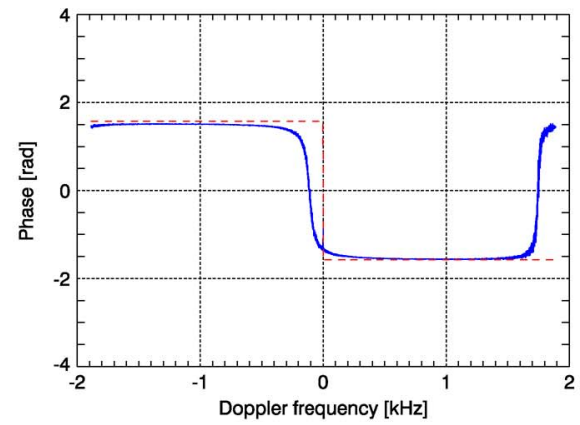

(b)

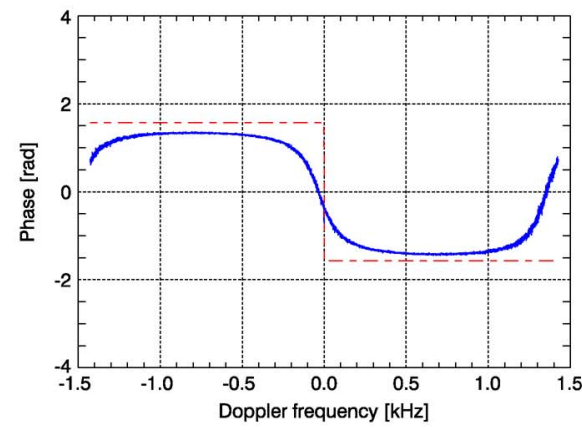

(e)

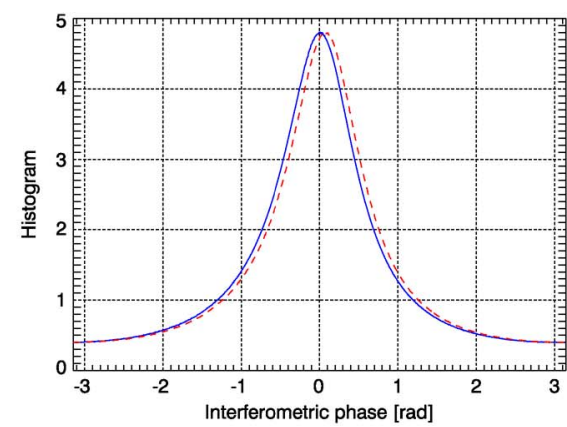

(c)

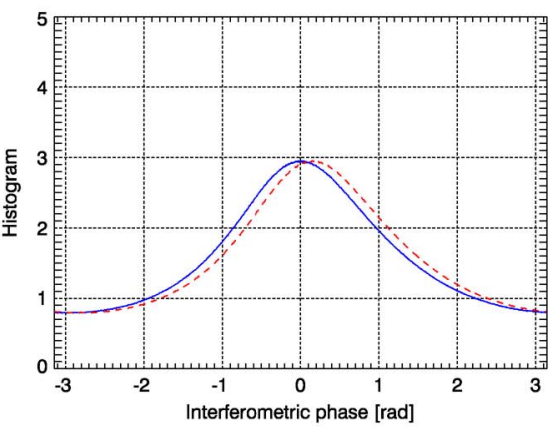

(f)

Fig. 6. Phase offset between the $\Sigma$ - and $\Delta$-channels: (a) and (d) before the calibration and (b) and (e) after the calibration (solid line: measured phase pattern, dashed line: reference phase pattern). (c) and (f) Phase imbalance between the fore-and aft-channels, estimated with the image data. The solid line indicates the phase histogram after the channel balancing, and the dashed line shows that before the balancing.

TABLE II

MeAsured OfFSETS AND CHANNEL IMBALANCE

\begin{tabular}{|c|c|c|c|}
\hline Channel & Error & DT1 & DT2 \\
\hline \multirow{2}{*}{$\Sigma-\Delta$} & phase offset & $110.42^{\circ}$ & $102.193^{\circ}$ \\
& amplitude offset & $0.53 \mathrm{~dB}$ & $0.33 \mathrm{~dB}$ \\
\hline \multirow{2}{*}{ fore - aft } & phase imbalance & $5.98^{\circ}$ & $8.79^{\circ}$ \\
& amplitude imbalance & $0.56 \mathrm{~dB}$ & $0.48 \mathrm{~dB}$ \\
\hline
\end{tabular}

offsets and imbalance. It is noted that the amplitude errors in Table II are average values since these are frequency dependent in real data.

\section{DBF SAR Imaging}

Using the fully calibrated signals, a digital beamformer, which is the reconstruction filter in this experiment, distinguishes original Doppler components from their periodic higher order spectral components (alias) at every Doppler bin [3] and reconstructs a Doppler spectrum. As a result, at the output of the digital beamformer, the signal within the Doppler bandwidth is reconstructed. The experimental SAR processor produces SAR images in the conventional way.

Normally, a SAR processor carries out not only the focusing but also additional signal processing steps, such as antenna pattern correction and an amplitude tapering for the adjustment of sidelobes of target impulse responses. However, in this experimental SAR processor, the pattern compensation and the tapering in azimuth are intentionally omitted, in order to prevent any enlargement or reduction in the ambiguity level of the final images.

First, Fig. 7 shows the images of DT2 in both the conventional single-channel mode and the DRA mode with DBF. For the given SAR parameters of this data take (see Table I), the first-order azimuth ambiguity is supposed to be displaced at approximately $4.07 \mathrm{~km}$ on both sides of the original target response according to [17]

$$
\Delta x_{\mathrm{az}} \approx \frac{f_{\mathrm{PRF}} \cdot \lambda \cdot R_{o}}{2 \cdot v_{\mathrm{eff}}^{2}} \cdot v_{g}
$$

where $\Delta x_{\mathrm{az}}$ is the azimuth displacement of the first-order ambiguity, $R_{o}$ is the minimum slant range, and $v_{\text {eff }}$ and $v_{g}$ are the effective velocity and the ground velocity, respectively. In Fig. 7(a), the azimuth ambiguities of the Maui island are clearly visible, owing to the very weak scattering of the sea clutter. On the other hand, the azimuth ambiguities are suppressed by the DBF in the DRA mode data, and thereby, the produced image presents a remarkable improvement in the ambiguity level for the same PRF and Doppler processing bandwidth. This result is presented in Fig. 7(b). As aforementioned, this ambiguity and its suppression can be depicted in the Doppler frequency spectrum domain. In Fig. 8, we plot the Doppler amplitude patterns of both images above.

According to the Nyquist and Shannon sampling theory [18], the Doppler spectrum is recovered within the PRF of the data take. However, due to the low PRF, this spectrum is interfered by the aliasing. This effect is more severe at higher frequency components. Fig. 8(a) shows the Doppler spectral amplitude pattern constructed from Fig. 7(a). In the DRA mode, the reconstruction filter removes the spectral aliasing and recovers the original baseband spectrum in the Doppler frequency range of [0, PRF] and [-PRF, 0], separately (see Fig. 4). Subsequently, these two spectra are concatenated to produce a wide Doppler spectrum. As a result, in Fig. 8(b), the Doppler frequency 


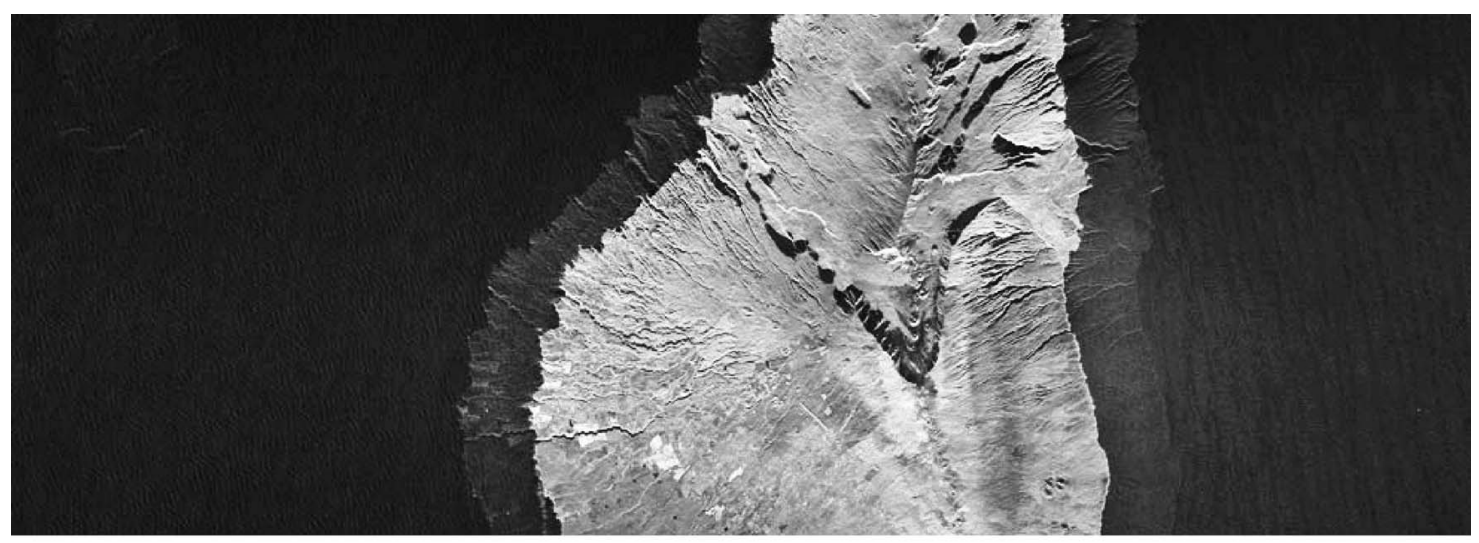

(a)

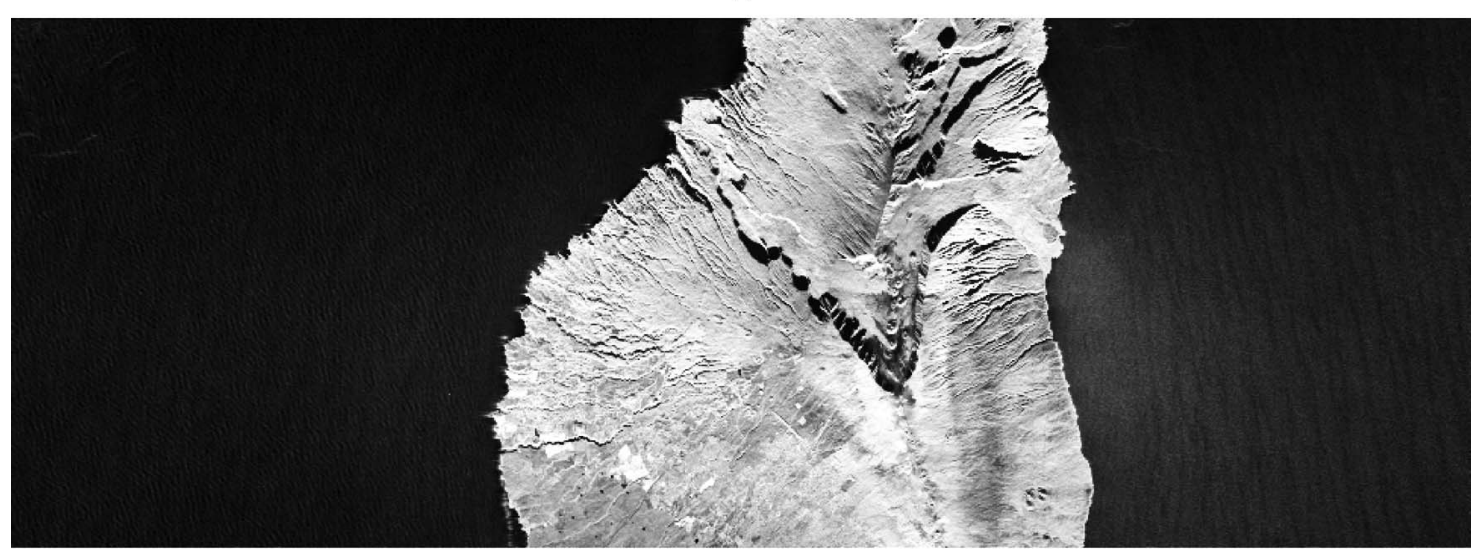

(b)

Fig. 7. TerraSAR-X images of DT2 over Hawaii, Maui island, USA, in the (a) conventional single-antenna mode and in the (b) DRA mode combined with DBF The vertical and horizontal axes are the range and azimuth direction, respectively. The Doppler processing bandwidth is set to $2.852 \mathrm{kHz}$ for both cases, and the antenna pattern correction and amplitude tapering are applied only in range.

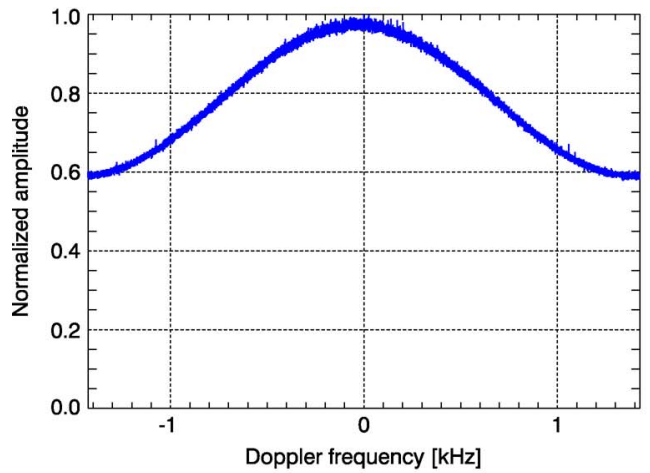

(a)

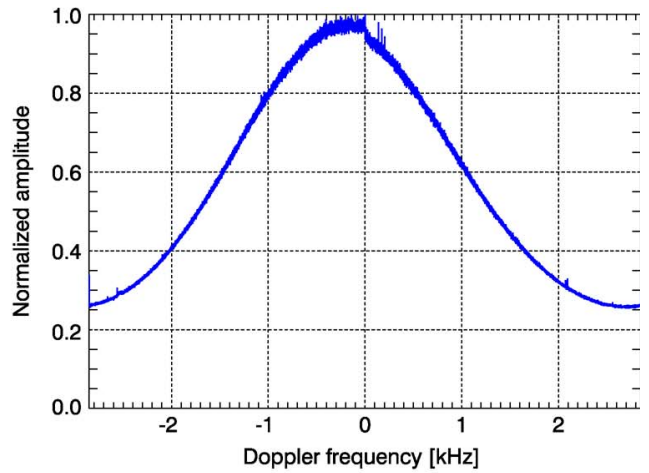

(b)

Fig. 8. Normalized Doppler amplitude pattern of DT2: (a) the conventional single-antenna mode and (b) the DRA mode with DBF.

range is expanded to the double PRF value. Since the Doppler amplitude pattern is equivalent to the azimuth antenna pattern [17], [19], this satisfactory recovery of the antenna pattern in Fig. 8(b) implicates the suppression of spectral aliasing, which is the direct cause of the azimuth ambiguity in the side-looking SAR.

In the same manner, the SAR images from DT1 are produced and presented in Fig. 9, and the Doppler amplitude patterns of each image are also plotted in Fig. 10. It is noted once again that the Doppler amplitude pattern in Fig. 10(b) is obtained in the Doppler range of $7.547 \mathrm{kHz}$, which is the effective PRF reconstructed by the DBF. Although the PRF of DT1 is higher than that of DT2, significant ambiguities are observed in the sea. This is because strong scatterers of artificial structures in the urban area are located along the coast, and consequently, their strong ambiguities plainly appear in the sea area as DT2. This situation can result in a serious disturbance for ship detection and surveillance, since targets close to the coast may not be properly discriminated from the strong ambiguities.

Let us assume that the intensity of a point target response is $40 \mathrm{~dB}$ higher than that of the sea clutter. Even in an image satisfying the $-25-\mathrm{dB}$ azimuth ambiguity-to-signal ratio 


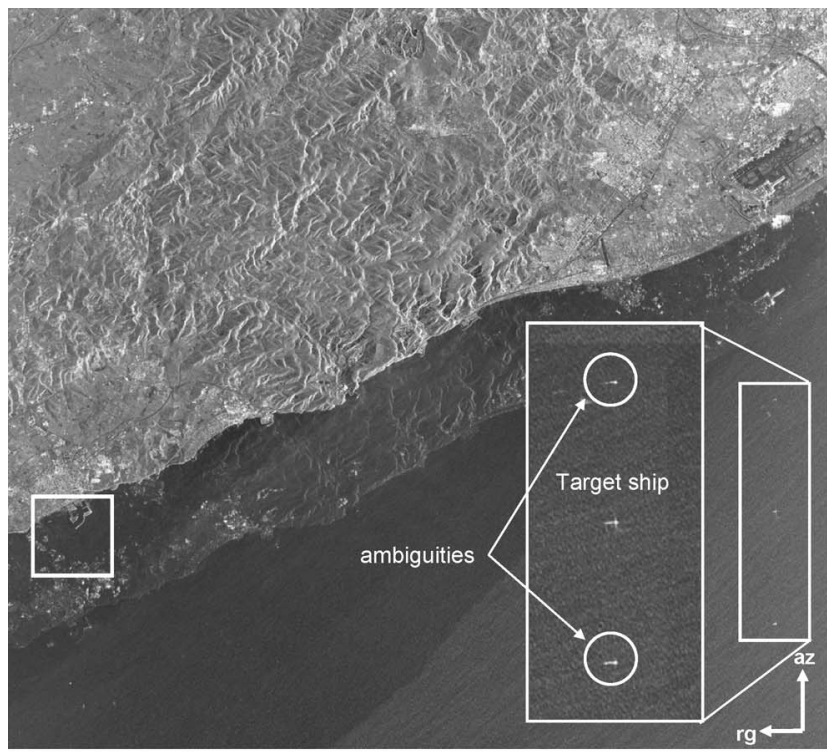

(a)

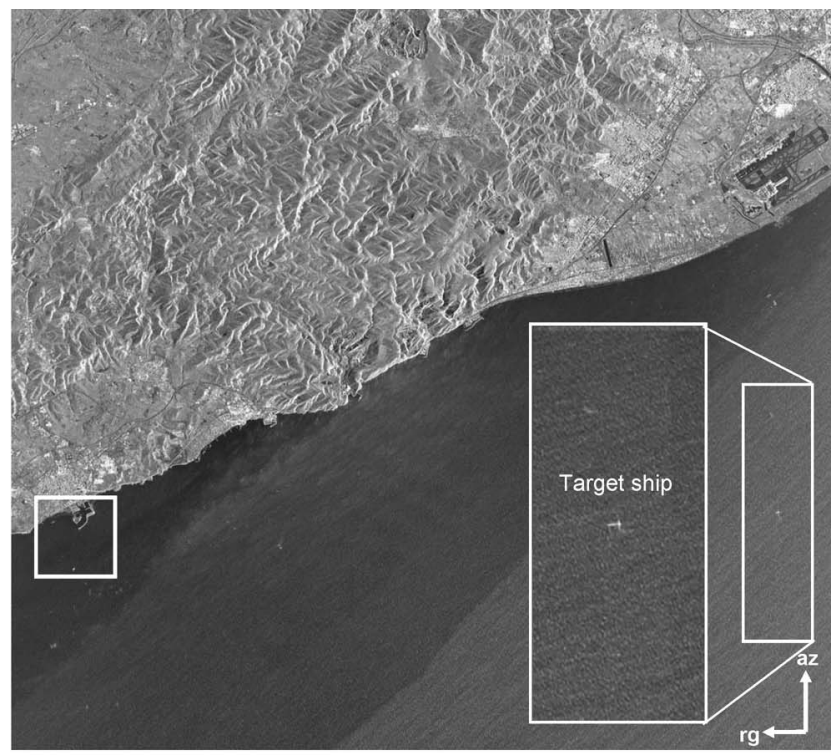

(b)

Fig. 9. TerraSAR-X images of DT1 over the region, Barcelona, Spain in the (a) conventional single-antenna mode and in the (b) DRA mode combined with DBF. The Doppler processing bandwidth is set to $3.773 \mathrm{kHz}$ for both cases, and the antenna pattern correction and amplitude tapering are applied only in range.

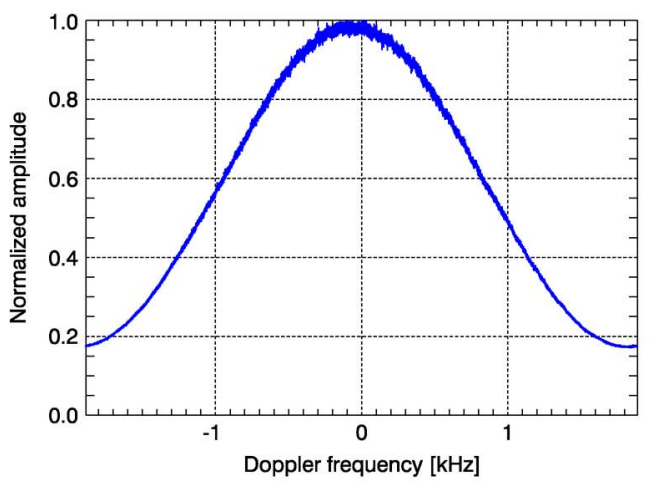

(a)

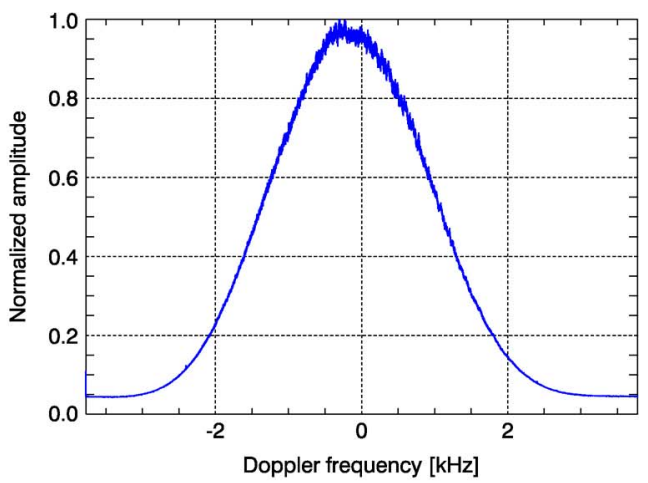

(b)

Fig. 10. Normalized Doppler amplitude pattern of DT1: (a) the conventional single-antenna mode and (b) the DRA mode with DBF.

(AASR), the ambiguity level of $-25 \mathrm{~dB}$ is $15 \mathrm{~dB}$ stronger than the sea clutter. In this case, the presence of ships being close to the coast cannot be properly detected. An example is shown in Fig. 11. A zoom over Vilanova i la Geltrú harbor, which is marked by the square in Fig. 9, is compared with the DBF image. The image by the single-channel mode is significantly disturbed by azimuth ambiguities of strong scatters in land, so that a ship (marked by the circle) is not recognized. When the ambiguity is removed from the image using DBF, the ship in the sea is detected, as shown in Fig. 11(b).

In Fig. 9, a ship being far away from the coast is selected for further investigations. The ship is imaged in the singleantenna mode, the data interleaving mode, ${ }^{2}$ and the DRA mode with DBF, respectively. Fig. 12 shows the main responses, their ambiguities, and the azimuth profiles at the maximum point of each image. It is obvious that there is a wide disparity in the ambiguity level. In particular, the difference between the interleaving mode and the DRA mode with DBF is worth

\footnotetext{
${ }^{2}$ This mode rearranges the fully calibrated azimuth data of the fore- and aftchannels in corresponding along-track positions, so that the multichannel data are transformed to single-channel data with a higher sampling rate.
}

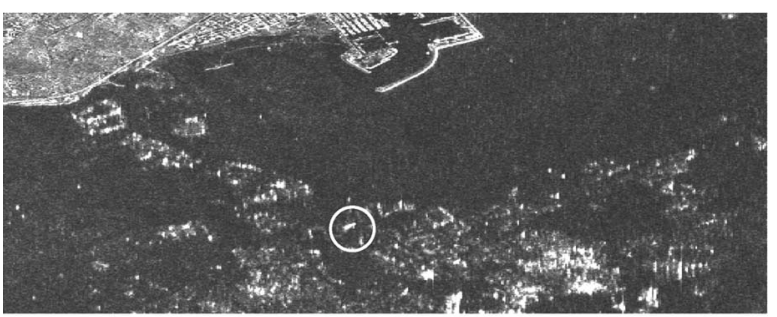

(a)

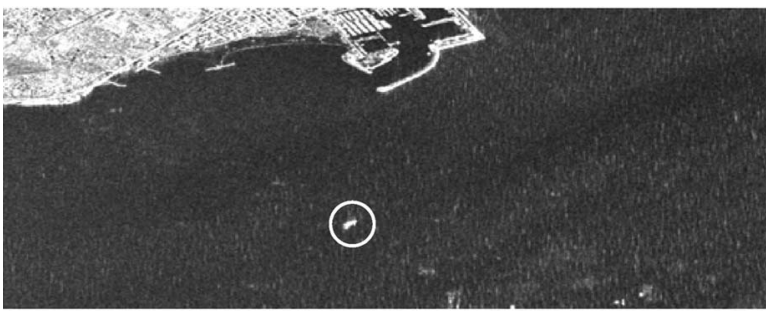

(b)

Fig. 11. Image comparison between the (a) conventional single-antenna mode and the (b) DRA mode with DBF with regard to ship detection. Due to strong ambiguities of targets in the coastal area, the ship in the circle is not distinguished. DBF removes the ambiguities without any loss of the target information (ship impulse response). 


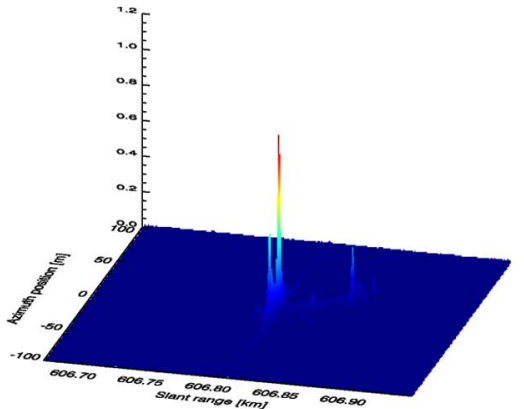

(a)

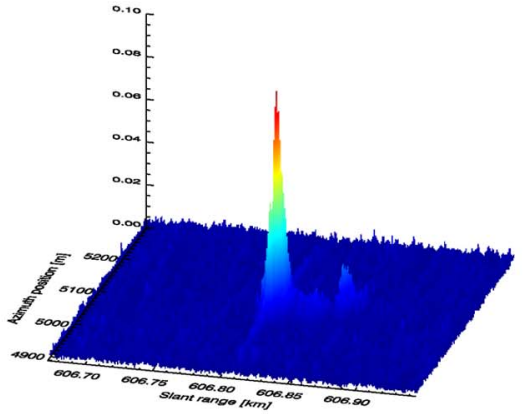

(d)

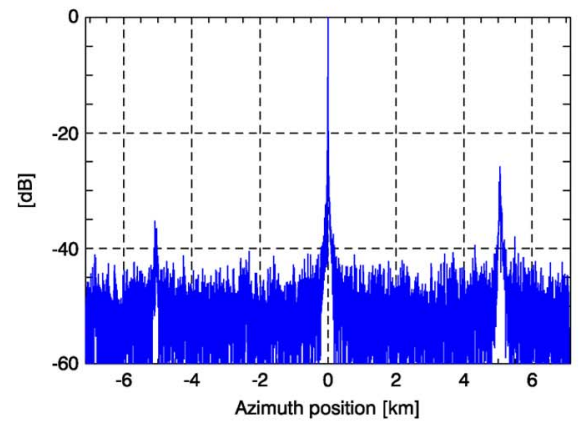

(g)

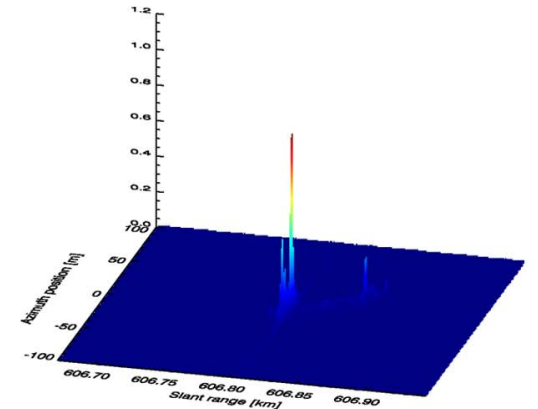

(b)

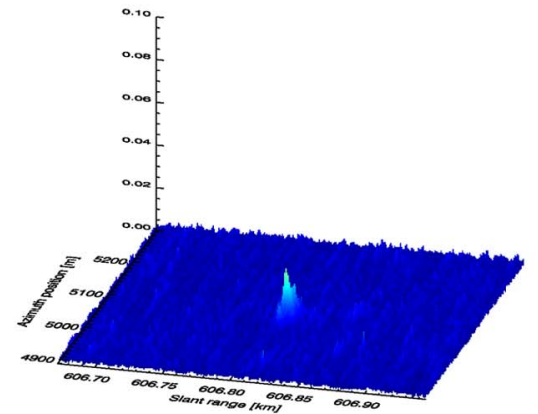

(e)

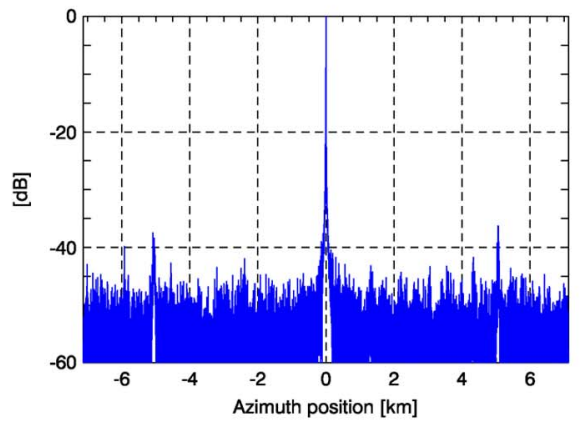

(h)

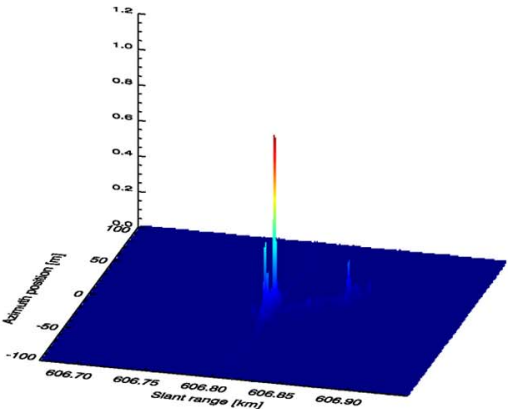

(c)

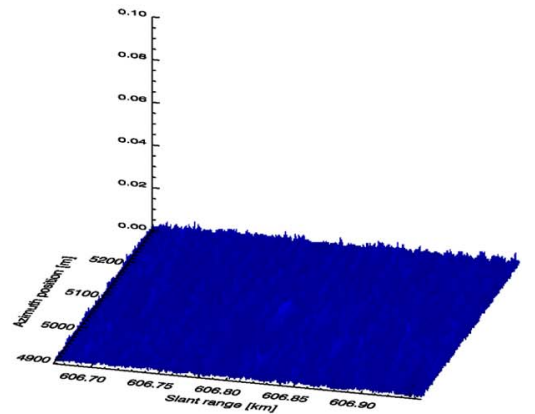

(f)

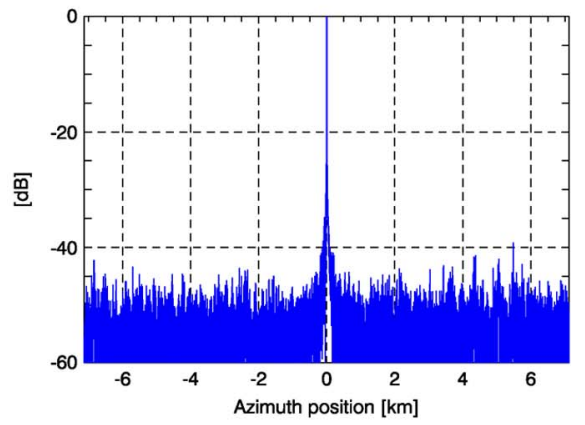

(i)

Fig. 12. First row exhibits the target impulse responses for the single-antenna mode, the interleaving mode, and the DRA mode with DBF. The second row shows the azimuth ambiguity of each response. The last row presents the azimuth profiles of each target response.

emphasizing [see Fig. 12(e) and (f)]. Although the data interleaving mode also increases the number of spatial samples, a phase mismatch due to the nonuniform samples is not corrected. As a result, residual ambiguities occur in the image. On the other hand, the DBF (reconstruction filter) compensates for a phase rotation caused by the nonuniform sampling. In consequence, both the images contain different levels of ambiguities with the same number of azimuth samples.

\section{DisCUSSION-AASR, AZIMUTH RESOLUTION, AND SNR}

According to the principle of high-resolution wide-swath (HRWS) imaging, small antennas for both the transmission and the reception are desired to acquire a wide Doppler band signal [20]-[22]. In the TerraSAR-X DRA mode, the full antenna aperture of $4.8 \mathrm{~m}$ is used for the transmission, so that a narrow beam $\left(0.33^{\circ}\right)$ in azimuth illuminates the area of interest. Therefore, we do not achieve a remarkable improvement in the spatial resolution in this experiment.

However, in regard to the application of the DBF to the ongoing mission, we can take a great advantage with respect to AASR, since DBF provides 15-dB AASR improvement for the same processed Doppler bandwidth. To reach the same level in the conventional mode, one needed to reduce the Doppler processing bandwidth to approximately $2.1 \mathrm{kHz}$. Then, a degradation of resolution is an inevitable consequence. In Fig. 13, the azimuth impulse response function processed with the $2.1 \mathrm{kHz}$ Doppler processing bandwidth is compared with that of the DBF SAR image. The azimuth resolution in the single-antenna mode is 1.63 times broader than that in the DRA mode with DBF.

Moreover, we consider the influence of the beamforming network on the signal-to-noise ratio (SNR) and introduce the SNR scaling factor $\Phi_{b f}$, which indicates SNR variation due to the beamforming network as a function of the PRF. This parameter is normalized by the optimum value for uniform sampling [15], i.e.,

$$
\Phi_{b f}=\frac{\sum_{n=1}^{N} E\left\{\left|P_{n}\left(f_{\mathrm{D}}\right)\right|^{2}\right\}}{N}
$$

where $P_{n}$ is the reconstruction filter consisting of $N$ subband functions [3], and $N$ is the number of receive channels. 


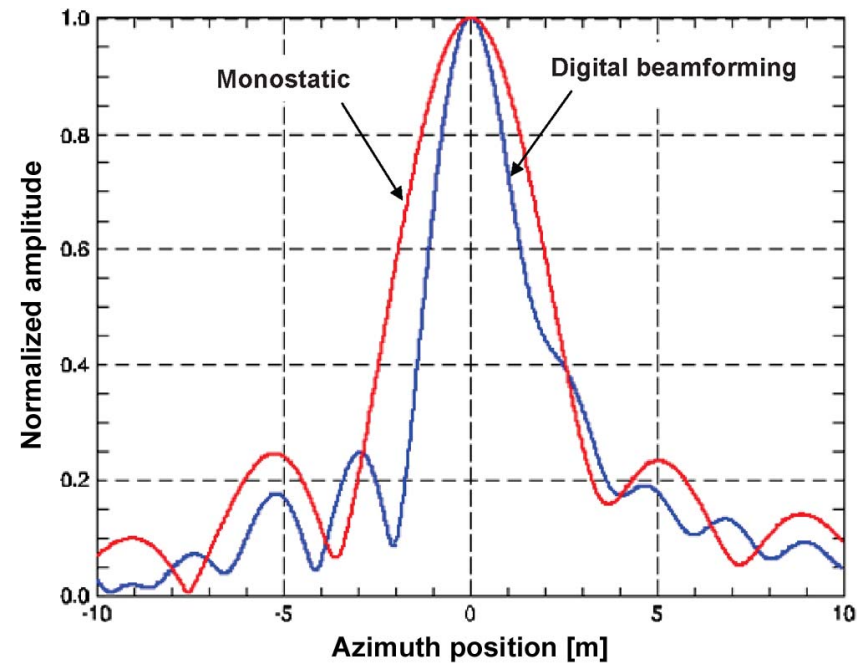

Fig. 13. Comparison of the azimuth resolution between the DBF SAR image and the conventional for the same azimuth ambiguity level.

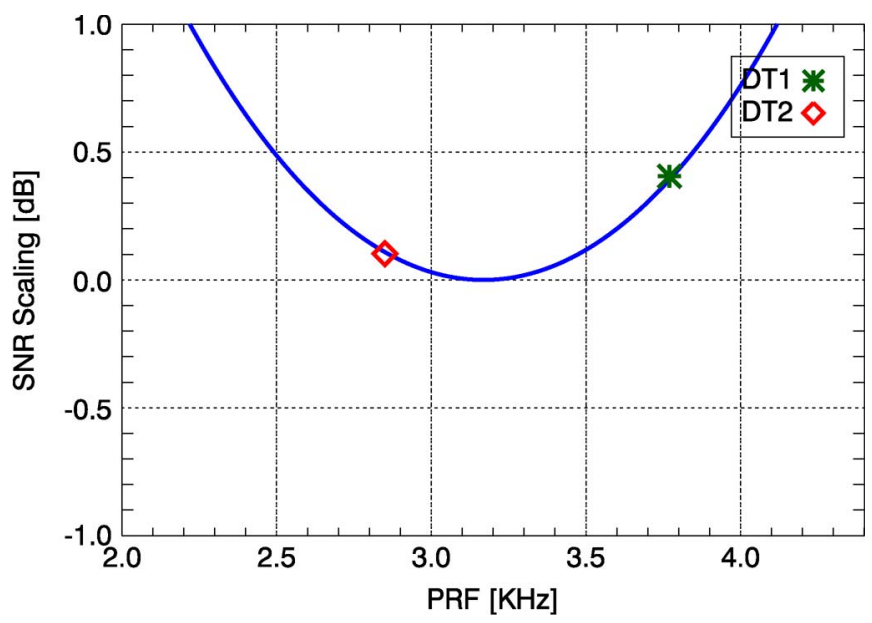

Fig. 14. Estimated SNR scaling factor for PRF variation.

Fig. 14 shows the estimated SNR scaling factor for the given TerraSAR-X DRA mode parameters, where the values corresponding to DT1 and DT2 are indicated by the asteroid and the diamond symbols, respectively.

The plot shows that the SNR scaling is proportional to the PRF offset from the uniform PRF. As stated in Table I, the uniformity of DT1 (118.4\%) is worse than DT2 (89.4\%), so that the SNR degradation of DT1 is approximately $0.3 \mathrm{~dB}$ higher than that of DT2. The AASR with regard to residual ambiguity energy after the DBF is estimated using the analytic model developed in [15] and is plotted in Fig. 15.

Although the exact ambiguity level of the acquired data takes is not measurable due to sea clutter noise, their approximated values match well to the model-based estimation. Therefore, the DRA mode with DBF will provide consistent AASR performance over a wide range of PRFs, for example, AASR lower than $-30 \mathrm{~dB}$ for a PRF $>2.2 \mathrm{kHz}$. According to the above discussion, it is possible to supply new products, which are improved either in the coverage or at the AASR level. Table III summarizes the specifications of the standard TerraSAR-X stripmap product and the two potential products.

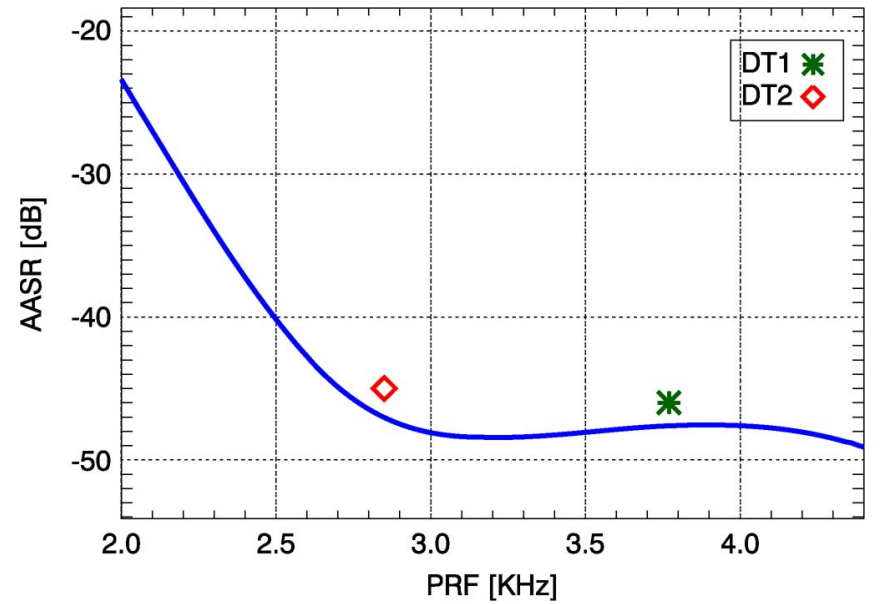

Fig. 15. (Solid line) Estimated AASR for PRF variation and measured AASR values.

TABLE III

Specifications of the Standard and the Potential Products

\begin{tabular}{|r|c|c|c|}
\hline & STD & Wide swath & Low AASR \\
\hline Imaging mode & \multicolumn{3}{|c|}{ Stripmap } \\
\hline Antenna mode & Single & DRA with DBF \\
\hline AASR [dB] & $<-17$ & $<-17$ & $<-30$ \\
\hline Swath width [km] & 30 & 50 & 30 \\
\hline Doppler BW [kHz] & 2.765 & 2.765 & 2.765 \\
\hline Azimuth resolution [m] & 3.0 & 3.0 & 3.0 \\
\hline
\end{tabular}

\section{CONCLUSION}

In this paper, we have presented the first spaceborne DBF experiment employing the TerraSAR-X DRA mode. Through this experiment, we have successfully demonstrated the ability of DBF to improve the AASR in a general spaceborne SAR scenario, which contains very complex physical effects and target characteristics. Therefore, this demonstration is of high importance for the implementation of future SAR systems, based on the HRWS imaging concept. Although the DBF function is not implemented in TerraSAR-X, all DBF processes, including the calibration and the channel balancing, could be realized by software on ground. That is a notable point showing the merit of DBF as well as its capability. Consequently, this experiment also shows the feasibility that the DBF can be applied to ongoing SAR missions, which employ the dual receive mode, in order to improve either the coverage or the ambiguity suppression. It allows more effective data acquisition, satisfying diverse demands of SAR users. In conclusion, it is worth emphasizing that the DBF is a beneficial technique not only for a future SAR system but also for ongoing spaceborne missions.

\section{REFERENCES}

[1] K. Tomiyasu, "Tutorial review of synthetic aperture radar (SAR) with applications to imaging of the ocean surface," Proc. IEEE, vol. 66, no. 5, pp. 563-583, May 1978.

[2] N. Gebert, G. Krieger, and A. Moreira, "Multichannel azimuth processing in ScanSAR and TOPS mode operation," IEEE Trans. Geosci. Remote Sens., vol. 48, no. 7, pp. 2994-3008, Jul. 2010.

[3] G. Krieger, N. Gebert, and A. Moreira, "Unambiguous SAR signal reconstruction from nonuniform displaced phase center sampling," IEEE Geosci. Remote Sens. Lett., vol. 1, no. 4, pp. 260-264, Oct. 2004.

[4] J. Kim, A. Ossowska, and W. Wiesbeck, "Experimental investigation of digital beamforming SAR performance using a ground-based demonstrator," in Proc. Int. Geosci. Remote Sens. Symp., Barcelona, Spain, Jul. 2007, pp. 111-114. 
[5] J. Kim, M. Younis, and W. Wiesbeck, "Experimental performance investigation of digital beamforming on synthetic aperture radar," in Proc. Int. Geosci. Remote Sens. Symp., Boston, MA, Jul. 2008, vol. 4, pp. 176-179.

[6] N. Gebert, F. Almeida, and G. Krieger, "Advanced multi-channel SAR imaging-Measured data demonstration," in Proc. Int. Radar Symp., Hamburg, Germany, Sep. 2009, pp. 525-529.

[7] N. Gebert, F. Almeida, and G. Krieger, "Airborne demonstration of multichannel SAR imaging," IEEE Geosci. Remote Sens. Lett., vol. 8, no. 5, pp. 963-967, Sep. 2011.

[8] German Aerospace Center (DLR) TerraSAR-X Mission Homepage. [Online]. Available: http://www.dlr.de/terrasar- $\mathrm{x}$

[9] J. Mittermayer and H. Runge, "Conceptual studies of exploiting the TerraSAR-X dual receive antenna," in Proc. Int. Geosci. Remote Sens. Symp., Toulouse, France, Jul. 2003, pp. 2140-2142.

[10] R. Romeiser and H. Runge, "Theoretical evaluation of several possible along-track InSAR modes of TerraSAR-X for ocean current measurements," IEEE Trans. Geosci. Remote Sens., vol. 45, no. 1, pp. 21-35, Jan. 2007.

[11] R. Romeiser, S. Suchandt, H. Runge, U. Steinbrecher, and S. Grunler, "First analysis of TerraSAR-X along-track InSAR-derived current fields," IEEE Trans. Geosci. Remote Sens., vol. 48, no. 2, pp. 820-829, Feb. 2010.

[12] P. Prats, M. Rodriguez-Cassola, L. Marotti, M. Nannini, S. Wollstadt, D. Schulz, N. Tous-Ramon, M. Younis, G. Krieger, and A. Reigber, "TAXI: A versatile processing chain for experimental TanDEM-X product evaluation," in Proc. Int. Geosci. Remote Sens. Symp., Honolulu, HI, Jul. 2010, pp. 4059-4062.

[13] M. Gabele, B. Bräutigam, D. Schulze, U. Steinbrecher, N. Tous-Ramon, and M. Younis, "Fore and Aft channel reconstruction in the TerraSAR-X dual receive antenna mode," IEEE Trans. Geosci. Remote Sens., vol. 48, no. 2, pp. 795-806, Feb. 2010.

[14] F. Q. de Almeida, "Multi-channel azimuth processing in SAR airborne measured data demonstration and analysis," M.S. thesis, DLR, ITA, Braunschweig, Germany, 2009.

[15] N. Gebert, G. Krieger, and A. Moreira, "Digital beamforming on receive: Techniques and optimization strategies for high-resolution wideswath SAR imaging," IEEE Trans. Aerosp. Electron. Syst., vol. 45, no. 2, pp. 564-592, Apr. 2009.

[16] C. Gierull, "Digital channel balancing of along-track interferometric SAR data," DRDC, Ottawa, ON, Canada, Tech. Rep. TM 2003-024, Mar. 2003.

[17] J. C. Curlander and R. N. McDonough, Synthetic Aperture Radar Systems and Signal Processing. Hoboken, NJ: Wiley, 1991.

[18] C. Shannon, "Communication in the presence of noise," Proc. Inst. Radio Eng., vol. 37, no. 1, pp. 10-21, Jan. 1949.

[19] I. G. Cumming and F. H. Wong, Digital Processing of Synthetic Aperture Radar Data. Norwood, MA: Artech House, 2005.

[20] G. Krieger and A. Moreira, "Potentials of digital beamforming in Bi- and multistatic SAR," in Proc. Int. Geosci. Remote Sens. Symp., Toulouse, France, Jul. 2003, vol. 1, pp. 527-529.

[21] M. Younis, C. Fischer, and W. Wiesbeck, "Digital beamforming in SAR systems," IEEE Trans. Geosci. Remote Sens., vol. 41, no. 7, pp. 17351739, Jul. 2003.

[22] M. Suess, B. Grafmueller, and R. Zahn, "A novel high resolution, wide swath SAR system," in Proc. IEEE Geosci. Remote Sens. Symp., Sidney, Australia, Jul. 2001, vol. 3, pp. 1013-1015.

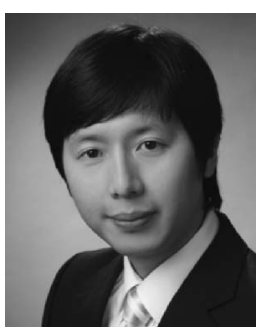

Jung-Hyo Kim (S'04-M'10) was born in Seoul, Korea, in 1972. He received the B.Sc. degree in space science and radio engineering from Kyunghee University, Seoul, in 1999 and the M.Sc. and Dr.Ing. (Ph.D.) degrees in electrical engineering from Yonsei University, Seoul, and the Karlsruhe Institute of Technology, Karlsruhe, Germany, in 2001 and 2011, respectively.

From 2001 to 2003, he was an RF System Engineer with LG Electronics, CDMA Laboratory, Seoul. From 2004 to 2009, he was a Research Scientist with the Institut für Hochfrequenztechnik und Elektronik, Universität Karlsruhe (TH). Since 2009, he has been with the Microwaves and Radar Institute, German Aerospace Center (DLR), Oberpfaffenhofen, Germany. His research interests include radar system engineering, advanced SAR techniques, such as digital beamforming and Orthogonal Frequency Division Multiplexing waveform design, and Multiple-Input Multiple-Output (MIMO) SAR systems.

Dr. Kim was the recipient of the second prize in the Student Paper Competition held during the IEEE International Geoscience and Remote Sensing Symposium 2008, Boston, MA.

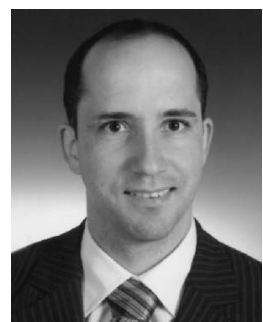

Marwan Younis (S'95-M'05-SM'08) was born in Las Cruces in 1970. He received the B.Sc. degree in electrical engineering from the University of Baghdad, Baghdad, Iraq, in 1992 and the Dipl.Ing. (M.Sc.) and Dr.-Ing. (Ph.D.) degrees in electrical engineering from the Universität Karlsruhe $(\mathrm{TH})$, Karlsruhe, Germany, in 1997 and 2004, respectively

From 1998 to 2004, he was a Research Scientist with the Institut für Hochfrequenztechnik und Elektronik, Universität Karlsruhe (TH). Since 2005, he has been with the Microwaves and Radar Institute, German Aerospace Center (DLR), Oberpfaffenhofen, Germany. He is a Lecturer with the Universität Karlsruhe (TH) and the Carl-Cranz-Gesellschaft. $\mathrm{He}$ is the author and coauthor of about 100 conference papers and more than 20 reviewed publications. His research fields include SAR systems, SAR performance, digital beamforming for radar, synchronization of bistatic SAR, forward looking radar, and antennas.

Dr. Younis is an active Member of the German Association for Location and Navigation (DGON). He is the active microwave working group leader within the IEEE instruments and future technologies technical committee and an Associate Editor of the IEEE Geoscience and Remote Sensing Letter. He received the Hermann-Billing award for his Ph.D. thesis in 2005.

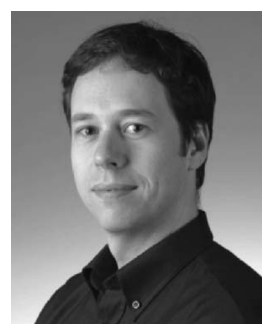

Pau Prats-Iraola (S'03-M'06) was born in Madrid, Spain, in 1977. He received the Ingeniero degree in telecommunication engineering and the Ph.D. degree from the Universitat Politècnica de Catalunya (UPC), Barcelona, Spain, in 2001 and 2006, respectively.

In 2001, he was with the Institute of Geomatics, Spain, as a Research Assistant designing a subaperture SAR processor. In 2002, he was with the Department of Signal Theory and Communications, UPC, where he worked in the field of airborne repeat-pass interferometry and airborne differential SAR interferometry. From December 2002 to August 2006, he was an Assistant Professor with the Department of Telecommunications and Systems Engineering, Universitat Autónoma de Barcelona, Barcelona. In 2006, he joined the Microwaves and Radar Institute, German Aerospace Center (DLR), Oberpfaffenhofen, Germany, where, since 2009, he has been Head of the Multimodal Algorithms Group. His research interests include air- and spaceborne SAR processing, SAR interferometry, differential SAR interferometry, and motion compensation for airborne systems.

Dr. Prats was the recipient of the first prize in the Student Paper Competition of the IEEE International Geoscience and Remote Sensing Symposium 2005 Conference held in Seoul, Korea.

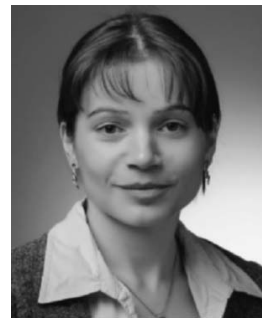

Martina Gabele received the Dipl.Ing. (M.Sc.) degree in electrical engineering from the Universität Karlsruhe (TH), Karlsruhe, Germany, in 2004. She was working toward the Ph.D. degree in the Microwaves and Radar Institute, German Aerospace Center (DLR), Oberpfaffenhofen, Germany, focusing on the area of system concepts and processing methods for spaceborne synthetic aperture radar (SAR) imaging and ground moving target indication (GMTI) from 2004 to 2011

Since 2004, she has been with the New SAR Missions Group and, since 2008, with the SAR Techniques Group of the Microwaves and Radar Institute, DLR. In 2006/2007, she was a Visiting Scientist with the Radar Systems Section of Defense R\&D Canada, Ottawa, ON, Canada. Since 2011, she has been working as a Consultant for radio and satellite systems with the IABG GmbH. 


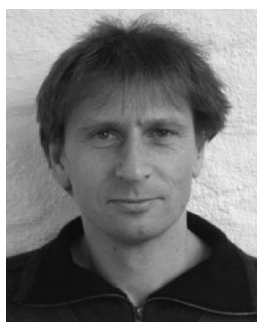

Gerhard Krieger (M'04-SM'09) received the Dipl.Ing. (M.S.) and Dr.-Ing. (Ph.D.) degrees (with honors) in electrical and communication engineering from the Technical University of Munich, München, Germany, in 1992 and 1999, respectively.

From 1992 to 1999, he was with the LudwigMaximilians University, Munich, where he conducted multidisciplinary research on neuronal modeling and nonlinear information processing in biological and technical vision systems. In 1999, he joined the Microwaves and Radar Institute (HR), German Aerospace Center (DLR), Oberpfaffenhofen, Germany, where he developed signal and image processing algorithms for a novel forward looking radar system employing digital beamforming on receive. From 2001 to 2007, he led the New SAR Missions Group, which pioneered the development of advanced bistatic and multistatic radar systems as exemplified by the forthcoming TanDEM-X mission as well as innovative multichannel SAR techniques and algorithms for high-resolution wide-swath SAR imaging. Since 2008, he has been Head of the new Radar Concepts Department of the Microwaves and Radar Institute, DLR. He is the author of more than 40 peer-reviewed journal papers, four invited book chapters, and about 250 conference papers. He is the holder of five patents. His current research interests focus on the development of multichannel radar techniques and algorithms for innovative MIMO SAR systems, the demonstration of novel interferometric and tomographic Earth observation applications, and the conceptual design of advanced bi- and multistatic radar missions.

Dr. Krieger received several national and international awards, including the W.R.G. Baker Prize Paper Award from the IEEE board of directors and the IEEE Transactions Prize Paper Award of the Geoscience and Remote Sensing Society. 